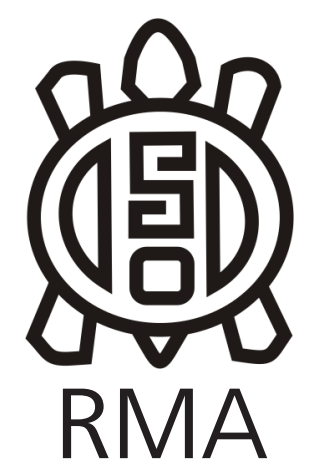

Dossier

\title{
Variaciones del terreno y cambios entesiales en poblaciones prehispánicas de Córdoba (Argentina)
}

\author{
Terrain variations and entheseal changes among prehispanic populations \\ from Córdoba (Argentina) \\ *Soledad Salega y *Mariana Fabra
}

*IDACOR-CONICET, Museo de Antropología, Facultad de Filosofía y Humanidades, Universidad Nacional de Córdoba, Argentina. E-mail: soledadsalega@gmail.com,

marianafabra@gmail.com

\begin{abstract}
Resumen
En años recientes se ha discutido si los cambios entesiales son sólo producto de movimientos físicos repetitivos o de actividades que generan sobrecarga. De acuerdo con Acosta et al. (2017) su manifestación en los miembros inferiores estaría influenciada por las características morfológicas del terreno, con una menor frecuencia de cambios entesiales entre los individuos de terrenos montañosos, debido a una mejor adaptación a las cargas biomecánicas. El objetivo del presente trabajo es evaluar la frecuencia de cambios entesiales para una muestra arqueológica (61 individuos) de las regiones de Sierras y Llanuras de la provincia de Córdoba (Argentina), aplicando dos metodologías de registro; a la vez que comparar estos resultados con los obtenidos previamente para cambios degenerativos de las articulaciones. Interesa, además, discutir los resultados en relación con la propuesta de Acosta et al. (2017). Se calcularon prevalencias porcentuales de la presencia de cambios en cuatro entesis de miembros inferiores y se realizaron tests estadísticos (Odds ratios, X2). Los resultados sugieren que la afectación sería algo mayor en Llanuras, aunque ello podría explicarse tanto desde un punto de vista adaptativo a la carga biomecánica, como desde una visión más clásica referida a la repetición de actividades físicas exigentes.
\end{abstract}

Palabras clave: actividad física; cambios entesiales;cambios degenerativos; Holoceno Tardío; Córdoba.

\begin{abstract}
Recently, it has been discussed whether the entheseal changes are only due to repetitive physical movements or activities that generate overload. According to Acosta et al. (2017) its manifestation would be influenced by the morphological characteristics of the terrain, with a lower frequency of entheseal changes among the individuals of mountainous terrain, due to a better adaptation to the biomechanical loads. The objective of the present work is to study the frequency of entheseal changes in an archaeological sample (61 individuals) from the Hills and Plains regions in the province of Córdoba (Argentina) by applying two recording methods; and to compare these results with those previously obtained when analyzing degenerative changes. The proposal of Acosta et al. (2017) is also discussed.Prevalences of entheseal changes in four entheses of the lower limb were calculated and statistical tests were performed (Odds ratios, X2). The results suggest that individuals were more affected in the Plains area, although this could be explained both by an adaptation to the biomechanical load or by a more classic view related to the repetition of demanding physical activities.
\end{abstract}

Keywords: physical activity; entheseal changes; degenerative changes; Late Holocene; Córdoba.

Una de las especialidades desarrolladas en el marco de la disciplina bioarqueológica pone su énfasis en el estudio de niveles de actividad física, como una manera de acercarse a la reconstrucción de los modos de vida de las poblaciones analizadas (Larsen 2002). Dos de los indicadores óseos utilizados para su análisis son los cambios degenerativos de las articulaciones y los cambios entesiales. Sin embargo, otros factores como la edad, el sexo, la masa corporal y la predisposición genética pueden relacionarse con estos cambios (Jurmain 2009; Jurmainet al. 2012; Villotte y Knüsel 2013). Además, la relación de estas modificaciones óseas con la actividad física aún no está claramente comprendida. Así, para el caso de los cambios entesiales, se ha discutido en años recientes si en todos los casos son producto de movimientos físicos repetitivos o de actividades que generen una cargabiomecánica (Alves Cardoso y Henderson 2013; Michopoulou et al. 2016; Milella et al. 2012). Específicamente, para los miembros inferiores, Acosta y colaboradores (2017) han sugerido que las características morfológicas del terreno tendrían influencia en la manifestación de estos cambios: se esperaría que su frecuencia fuera menor en los individuos que habitaron terrenos montañosos, ya que tendrían una mejor adaptación a las cargas biomecánicas y por lo tanto una menor sobrecarga(Henderson 2013). 
Trabajos anteriores realizados en el actual territorio de la provincia de Córdoba (Argentina) relevando cambios degenerativos de las articulaciones y nódulos de Schmorl han demostrado que existen diferencias entre los individuos provenientes de sectores llanos y de montañas. Mientras los primeros muestran lesiones degenerativas desde los primeros años de adultez hasta edades avanzadas, entre los pertenecientes a sectores

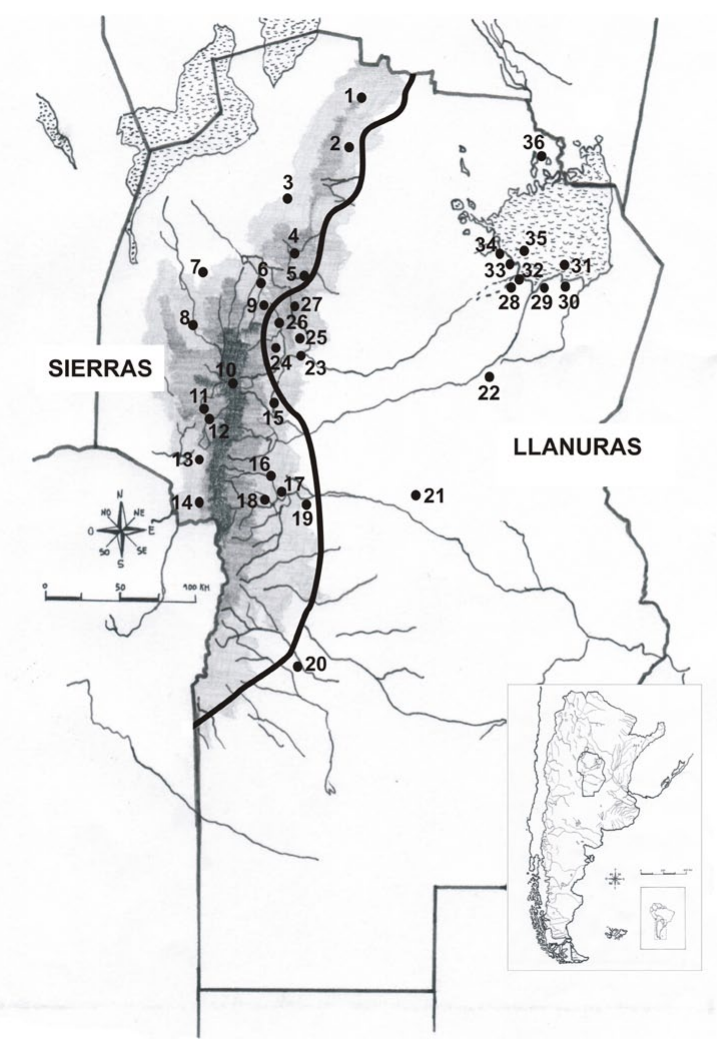

Figura 1.Ubicación de los sitios arqueológicos en las regiones de Sierras y Llanuras. 1) Estancia El Jume; 2) Cerro Colorado-S4; 3) El Estanque; Ischilín; 4) Nunsacat; 5) El Vado; 6) San Esteban; 7) Dorrego - Villa de Soto; 8) Rosca Yaco; 9) Ayampitín; 10) Copina; 11) Paraje La Cuesta; 12) Cañada Larga; 13) Guasmara; 14) Loma Bola; La Paz; 15) Constantinopla 1215; Potrerillo de Larreta; 16) Loteo 5 - Santa Rosa; 17) Potrero de Garay; 18) Amboy; 19) Mosconi - Río Tercero; 20) Río Cuarto - Barrio Alberdi; 21) Tío Pujio - James Craik; 22) Arroyito - Campo Sismondi; 23) Los Cielos; 24) Ecoterra; Cosquín 1977; 25) La Calera; Cabana; 26) Candonga; 27) La Granja; 28) Laguna del Plata; 29) Colonia Müller; 30) Orihuela; La Orihuela (Orih 08); 31) Isla Orihuela - Playa Sudeste; 32) Los Surgentes 1995; 33) El Diquecito; 34) Estancia La Elisa 2009; Campo Bocassi - Agua Mansa; La Para 015; 35) El Mistolar; 36) La Rinconada - Río Seco.

Figure1. Location of archaeological sites in the Hills and Plains regions. 1) Estancia El Jume; 2) Cerro Colorado-S4; 3) El Estanque; Ischilín; 4) Nunsacat; 5) El Vado; 6) San Esteban; 7) Dorrego - Villa de Soto; 8) Rosca Yaco; 9) Ayampitín; 10) Copina; 11) Paraje La Cuesta; 12) Cañada Larga; 13) Guasmara; 14) Loma Bola; La Paz; 15) Constantinopla 1215; Potrerillo de Larreta; 16) Loteo 5 - Santa Rosa; 17) Potrero de Garay; 18) Amboy; 19) Mosconi - Río Tercero; 20) Río Cuarto - Barrio Alberdi; 21) Tío Pujio - James Craik; 22) Arroyito - Campo Sismondi; 23) Los Cielos; 24) Ecoterra; Cosquín 1977; 25) La Calera; Cabana; 26) Candonga; 27) La Granja; 28) Laguna del Plata; 29) Colonia Müller; 30) Orihuela; La Orihuela (Orih 08); 31) Isla Orihuela - Playa Sudeste; 32) Los Surgentes 1995; 33) El Diquecito; 34) Estancia La Elisa 2009; Campo Bocassi - Agua Mansa; La Para 015; 35) El Mistolar; 36) La Rinconada - Río Seco. montañosos hay una baja afectación en el grupo de adultos jóvenes, aumentando luego entre los adultos medios (Salega y Fabra 2013).

Por ello, en el presente trabajo, nos proponemos evaluar la frecuencia de cambios entesiales en una muestra arqueológica proveniente de Córdoba, así como comparar los resultados obtenidos para ambos tipos de marcadores. Nos interesa, además, discutir los resultados en relación con la propuesta de Acosta et al. (2017). Por otra parte, nos proponemos utilizar dos metodologías de registro de cambios entesiales y evaluar si existen diferencias entre los resultados generados por ambas.

\section{Material y métodos}

La muestra analizada está compuesta por 61 individuos adultos, provenientes de la región serrana (30 individuos) y de llanura (31 individuos) del actual territorio de la provincia de Córdoba (Argentina), ubicados cronológicamente en el Holoceno Tardío (Figura 1). El material proviene tanto de excavaciones de rescate como de colecciones de museos de la provincia.

La determinación del sexo se realizó siguiendo los procedimientos compilados por Buikstra y Ubelaker (1994). Se tuvieron en cuenta las características morfológicas de la pelvis (arco ventral, concavidad subpúbica, rama isquiopúbica, escotadura ciática mayor, surco preauricular) y el cráneo (cresta nucal, proceso mastoideo, margen supraorbital, glabela, eminencia mental). Para la estimación de edad, se consideró la morfología de la sínfisis púbica (Brooks y Suchey 1990) y la superficie auricular del ilion (Lovejoy et al. 1985).

En cuanto al registro de los cambios entesiales, se consideraron las entesis de los músculos semimembranoso, glúteo medio, tendón patelar y tríceps sural, en ambas lateralidades. Para su registro se utilizó el método propuesto por Hawkey y Merbs (1995) y el nuevo método Coimbra (Henderson et al. 2016). En el primero, se distinguen tres categorías de cambios entesiales (robusticidad, lesiones por estrés y exostosis), para cada una de las cuales se registran tres grados de desarrollo. La elección de este método se debe a que ha sido el más utilizado hasta el momento. Sin embargo, ya que no distingue anatómicamente entre entesis fibrosas y fibrocartilaginosas y no da cuenta del efecto de la edad, se aplicó además el segundo método.

El nuevo método Coimbra distingue dos zonas anatómicas en cada entesis y registra seis tipos de cambios entesiales: formación ósea y erosión para zona 1 y zona 2; y cambio textural, porosidad fina, macroporosidad y cavitaciones para la zona 2. Para cada uno de estos cambios se distinguen dos grados, con excepción del cambio textural, para el que sólo registra su presencia (Figura 2).Este método, de reciente 


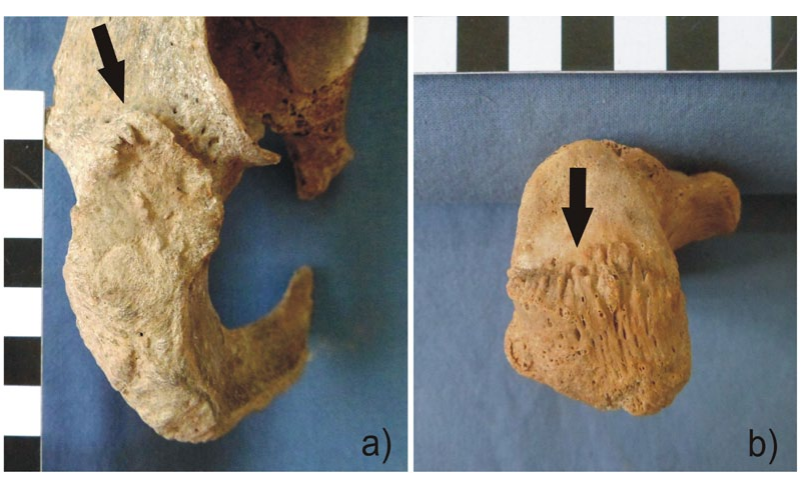

Figura 2. Ejemplos de cambios entesiales (nuevo método CoimbraHenderson et al. 2016-). Formación ósea en zona 1 en entesis de a) semimembranoso, y b) tríceps sural.

Figure 2. Example of entheseal changes (new Coimbra method -Henderson et al. 2016-). Bone formation in zone 1 of the entheses of a) semimembranosus, and b) triceps surae.

desarrollo, presenta las ventajas de ser biológicamente apropiado para las entesis de tipo fibrocartilaginosas, así como de permitir el registro de los distintos rasgos de manera separada en las distintas zonas de cada entesis (Henderson et al. 2013, 2016).

Para su análisis, las muestras se agruparon de acuerdo con la edad (adultos jóvenes -18 a 34 años de edady adultos medios -35 a 50 años-), y la procedencia geográfica (Sierras y Llanuras). Posteriormente, se calcularon las frecuencias para cada uno de los rasgos registrados en cada entesis, y finalmente se realizaron los análisis estadísticos de razón de probabilidades (Odds ratios) y de Chi cuadrado (X2) para establecer si las diferencias identificadas a partir de las prevalencias resultaban estadísticamente significativas. El nivel de significación utilizado fue de $p \leq 0,05$.

\section{Resultados}

La división de la muestra por sexo y edad resultó en 21 masculinos (14 adultos jóvenes y 7 adultos medios) y 9 individuos femeninos (3 adultos jóvenes y 6 adultos medios) para la zona de Sierras. Por su parte, en la zona de Llanuras, se registraron 17 masculinos (12 adultos jóvenes y 5 adultos medios) y 14 individuos femeninos ( 5 adultos jóvenes y 9 adultos medios).

El registro de los cambios entesiales de acuerdo con el método de Hawkey y Merbs (1995) sólo dio valores para los tres grados correspondientes a la categoría de robusticidad, es decir que no se registraron cambios asociados con las de lesión y exostosis. Por otra parte, al aplicar el nuevo método Coimbra sí se registraron valores correspondientes a los dos grados, pero con frecuencias muy bajas para el grado 2 .

Tabla 1. Presencia de cambios entesiales por procedencia geográfica y edad (método de Hawkey y Merbs 1995). N=total de entesis observadas; $\mathrm{C}=$ frecuencias de entesis con cambios, expresadas en porcentajes.

Table 1. Presence of entheseal changes according to geographical origin and age (Hawkey \& Merbs 1995's method). N=total of observed entheses; $C=$ frequencies of entheses with changes, in percentages.

\begin{tabular}{lccccccccc}
\hline & \multicolumn{4}{c}{ Sierras } & \multicolumn{4}{c}{ Llanuras } \\
& \multicolumn{2}{c}{ jóvenes } & \multicolumn{2}{c}{ medios } & \multicolumn{2}{c}{ jóvenes } & \multicolumn{2}{c}{ medios } \\
& N & C & N & C & N & C & N & C \\
\hline Semimembranoso & 22 & 95.5 & 11 & 100 & 17 & 94.1 & 21 & 100 \\
Glúteo medio & 17 & 82.4 & 14 & 85.7 & 19 & 63.2 & 15 & 66.7 \\
Tendón patelar & 22 & 95.5 & 15 & 86.7 & 22 & 90.9 & 21 & 71.4 \\
Tríceps sural & 10 & 70 & 15 & 93.3 & 18 & 100 & 15 & 100 \\
\hline
\end{tabular}

En trabajos previos, también se ha detectado esta baja frecuencia, lo cual dificultó su inclusión en los análisis estadísticos (Henderson et al. 2013). Por ello, y para posibilitar la comparación entre los resultados de ambos métodos, se decidió considerar sólo la presencia de cambios entesiales.

De acuerdo con el método de Hawkey y Merbs (1995), las frecuencias de dichos cambios superan en todos los casos el $60 \%$, aunque como ya fue mencionado, todos refieren a la categoría robusticidad. Las diferencias entre grupos de edad no son marcadas, mientras que entre ambas regiones se observan valores semejantes, cinco son mayores en Sierras y dos en Llanuras (Tabla 1).

Teniendo en cuenta el método de Henderson et al. (2016), en general para ambas regiones todas las entesis registran algún tipo de cambio y predominan la formación ósea en ambas zonas, seguida por la erosión en zona 2. Entre los individuos jóvenes de Sierras sólo aparecen valores mayores a $20 \%$ en semimembranoso y tendón patelar, mientras que entre los adultos medios esto se extiende al resto de las entesis. Por su parte, si consideramos a la región de Llanuras, hay una mayor cantidad de entesis con frecuencias mayores a $20 \%$ si

Tabla 2. Presencia de cambios entesiales por procedencia geográfica y edad (nuevo método Coimbra-Henderson et al. 2016-). N1=total de entesis observadas en Zona 1; N2=total de entesis observadas en Zona 2; FO: formación ósea; ER: erosión; CT: cambio textural; MIP: microporosidad. Las frecuencias están expresadas en porcentajes.

Table 2. Presence of entheseal changes according to geographical origin and age (new Coimbra method-Henderson et al. 2016-). N1=total of observed entheses in Zone 1; N2=total of observed entheses in Zone 2; FO: bone formation; ER: erosion; CT: textural change;MIP: microporosity. Frequencies are expresed in percentages.

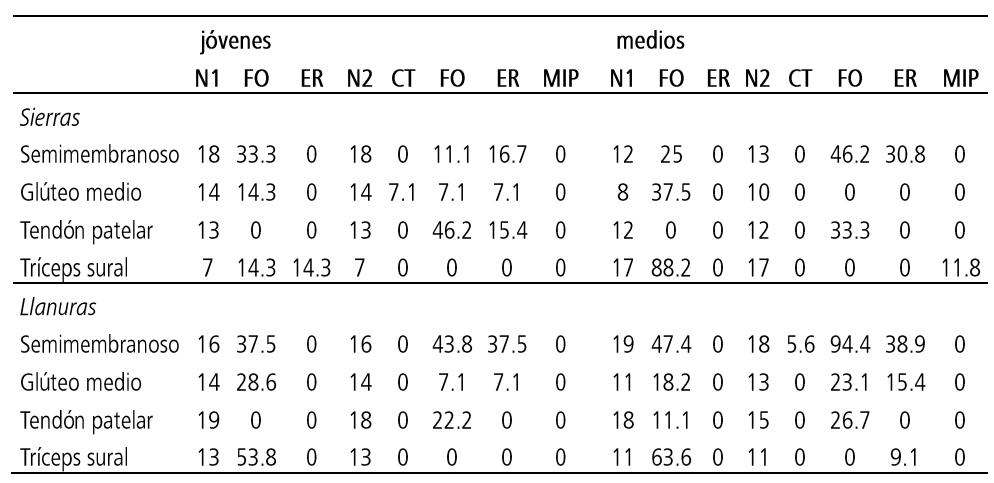


los comparamos con el mismo grupo etario de Sierras. Para los adultos medios, la cantidad de valores mayores a $20 \%$ es igual en ambas regiones, pero en Llanuras se registra una mayor cantidad de rasgos (Tabla 2).

El análisis estadístico aplicado a la totalidad de la muestra, sólo diferenciando su procedencia geográfica, no arrojó resultados significativos. Por otro lado, al distinguir a su vez entre individuos jóvenes y medios, se registraron 11 valores mayores a 2 para el cálculo de razón de probabilidades (Odds ratios). Para ambos métodos de registro, estos valores se asocian con las entesis del semimembranoso, el glúteo medio y el tríceps sural, a los que se agrega el tendón patelar para el nuevo método Coimbra. Sólo tres resultaron estadísticamente significativos de acuerdo con el test de X2 (Tabla 3) y corresponden a la región de Llanuras.

\section{Discusión y conclusiones}

Desde un punto de vista biológico y adaptativo, Acosta y colaboradores (2017) proponen que el desarrollo de los cambios entesiales está en relación con los niveles de adaptación músculo-esqueletal a las cargas a las que es sometido un individuo a lo largo de su vida, entonces una buena adaptación biomecánica implicará una menor frecuencia en la aparición de cambios entesiales (Henderson 2013). Por lo tanto, el desarrollo de éstos podría entenderse como el resultado de una sobrecarga a los límites fisiológicos. Teniendo en consideración que los habitantes de terrenos montañosos poseen una mejor adaptación fisiológica a ese ambiente, entonces se sigue que presentarían frecuencias de cambios entesiales menores que aquellos individuos procedentes de terrenos llanos. El resultado de su meta-análisis apoya esta interpretación para lasentesis del trocánter mayor, tendón patelar y tríceps suralen el caso de las mujeres, mientras que para los hombres algunos valores se asocian a terrenos montañosos (tríceps sural) y otros a planicies (trocánter mayor).

Para nuestro caso de estudio, no identificamos una tendencia distintiva desde el cálculo de frecuencias. Si consideramos el método de Hawkey y Merbs (1995), los valores correspondientes a los miembros inferiores son similares entre ambas regiones, mientras que si consideramos al método de Henderson et al. (2016) las frecuencias más altas en Llanuras se registran en el semimembranoso y glúteo medio, pero en Sierras hay también valores mayores como en el caso del tríceps sural entre los adultos medios. Sin embargo, desde los análisis estadísticos, los valores significativos sí se asocian con la región de Llanuras. Por otra parte, al sumar los resultados referidos a los cambios degenerativos (Salega 2016; Salega y Fabra 2013) se observa en general una mayor afectación en los individuos provenientes de Llanuras, con mayor cantidad de porciones anatómicas comprometidas, lo cual también es válido para el resto del esqueleto apendicular y para la columna vertebral. Si tenemos en cuenta entonces estos resultados y los provenientes del X2, podríamos acordar con la hipótesis de Acosta et al. (2017) para explicar la mayor frecuencia de cambios en la zona de Llanuras.

Por otro lado, y desde una interpretación más clásica, la mayor presencia de cambios degenerativos en los miembros inferiores y el sector bajo de la columna, así como un mayor desarrollo de cambios entesiales en miembros inferiores se han relacionado con el desplazamiento a pie en terrenos abruptos y sinuosos, combinado con el traslado de cargas (al-Oumaoui et al. 2004; Arrieta y Mendonça 2011; Lieverseet al. 2007, 2013;Quevedo Kawasaki 2000). Si retomamos esta interpretación, podríamos sugerir que los individuos procedentes de la región de Llanuras se vieron más exigidos durante la realización de las actividades cotidianas, que sus pares de las Sierras. En principio parecería contradictorio según esta interpretación. Sin embargo, si tenemos en cuenta las características ambientales de la región serrana, las diferencias altitudinales determinan la formación de pisos ecológicos con especies vegetales y animales adaptadas a las condiciones de precipitaciones y suelos propias de cada piso, permitiendo el aprovechamiento de una mayor variedad de recursos y en diferentes épocas del año, tanto de productos recolectados (Laguens y Bonnin 2009;

\begin{tabular}{lcccccc}
\hline & $\mathrm{n} / \mathrm{N}$ & $\%$ & odds ratios & $\mathrm{x} 2$ & $\mathrm{gl}$ & $\mathrm{p}$ \\
\hline $\begin{array}{l}\text { Hawkey y Merbs (1995) } \\
\text { Jóvenes }\end{array}$ & & & & & & \\
Tríceps sural D & $23 / 32$ & 71,9 & 3,857 & 3,239 & 1 & 0,072 \\
$\begin{array}{l}\text { Tríceps sural I } \\
\text { Medios }\end{array}$ & $12 / 32$ & 37,5 & 5,571 & $\mathbf{4 , 8 0 0}$ & $\mathbf{1}$ & $\mathbf{0 , 0 2 8}$ \\
Semimembranoso I & $15 / 28$ & 46,9 & 6,188 & 5,073 & 1 & 0,024 \\
Glúteo medio I & $8 / 28$ & 25 & 3,667 & 2,068 & 1 & 0,150 \\
\hline $\begin{array}{l}\text { Henderson et al. (2016) } \\
\text { Jóvenes }\end{array}$ & & & & & & \\
Semimembranoso D & $8 / 28$ & 25 & 2,500 & 1,163 & 1 & 0,281 \\
Tríceps sural D & $4 / 28$ & 12,5 & 4,200 & 1,532 & 1 & 0,216 \\
Tríceps sural I & $5 / 28$ & 15,6 & 6,222 & 2,758 & 1 & 0,097 \\
Medios & & & & & & \\
Semimembranoso D & $12 / 23$ & 37,5 & 5,333 & 3,569 & 1 & 0,059 \\
Semimembranoso I & $14 / 23$ & 43,7 & 20,000 & 7,987 & 1 & 0,005 \\
Glúteo medio I & $3 / 23$ & 9,4 & 2,444 & 0,491 & 1 & 0,484 \\
Tendón patelar I & $4 / 23$ & 12,5 & 4,125 & 1,433 & 1 & 0,231 \\
\hline
\end{tabular}

Tabla 3. Resultados de los tests de razón de probabilidades y Chi-cuadrado, de acuerdo con la procedencia geográfica (Sierras y Llanuras) y la edad. Se presentan los valores de Odds ratios mayores a 2, y en negrita los estadísticamente significativos ( $\mathrm{p} \leq 0.05)$. $\mathrm{n} / \mathrm{N}=$ total de entesis con cambios/ total de entesis relevadas; \%=porcentaje de afectación; $\mathrm{gl}=$ grado de libertad; $\mathrm{p}=$ significancia estadística.

Table 3. Results of Odds ratios and Chi-square tests, according to geographical origin (Hills and Plains), and age. Odds ratios values greater than 2 are presented, and statistical significant values $(p \leq 0.05)$ are in bold. $n / N=$ total of entheses with changes/total of recorded entheses; $\%=$ percentage of affected entheses; $g l=$ degree of freedom; $p=$ statistical significance. 
Medina et al. 2016) como de productos cultivados (Pastor y Berberián 2007; Pastor y López 2010), recursos de caza (Medina y Pastor 2012) y materias primas. A ello debemos agregar además la distancia relativamente reducida entre los sitios residenciales y los diferentes pisos ecológicos, con la consecuencia de que el costo energético de adquisición de recursos es bajo (Laguens 1999) y por lo tanto las jornadas dedicadas al traslado y las distancias se habrían visto disminuidas.

Por su parte, en las Llanuras los asentamientos se encuentran en ambientes más bien homogéneos, con menor diferencia altitudinal, lo cual sugeriría una menor variabilidad ambiental. Si bien la ubicación de los sitios podría haber aprovechado el potencial brindado por la fauna acuática y la vegetación ribereña (Laguens y Bonnin 2009), aún no se han realizado estudios acerca de la eficiencia de las estrategias de subsistencia en esta región, y desde el registro arqueológico no se ha determinado el uso efectivo de estos recursos (ibídem). Sin embargo, la adquisición de otro tipo de recursos habría implicado costos energéticos relativamente altos, por ejemplo materias primas como cuarzo, cuarcita de grano fino y ópalo (Bonofiglio et al. 1982), o bienes de procedencia extra regional, como bivalvos (Fabra et al. 2012; Fabra y Gordillo 2014). A partir de esto, podemos pensar que los grupos que vivieron en la zona de Llanuras debieron trasladarse a través de distancias mayores para procurarse productos que su propia región no les brindaba, o valerse de estrategias de intercambio con grupos asentados en sitios próximos a las fuentes de aprovisionamiento de dichos productos o materias primas.

Consideramos entonces que los cambios entesiales relevados en los miembros inferiores de nuestra muestra pueden ser explicados tanto desde un punto de vista adaptativo a la carga biomecánica, como desde una visión más clásica referida a la repetición de actividades físicas exigentes. Sin embargo, también se debe tener en cuenta, como limitación, que la muestra analizada es pequeña, por lo que los resultados pueden estar sobrestimando o subestimando las frecuencias reales de la población. Por otra parte, es necesaria una mayor cantidad de estudios que apliquen un método biológicamente apropiado (como en este caso, el nuevo método Coimbra para las entesis fibrocartilaginosas), para así poder establecer comparaciones con un mayor nivel de confiabilidad.

Córdoba, 9 de abril de 2017

\section{Agradecimientos}

Las autoras desean agradecer en primer lugar a los museos que albergan las colecciones relevadas en este trabajo. $Y$ en segundo lugar, a los comentarios de los evaluadores, por las observaciones realizadas. Este trabajo forma parte del proyecto "Estudios bioarqueológicos en poblaciones de la región central de Argentina" (PICT 2013-2028).

\section{Bibliografía}

Acosta, M. A., C. Y. Henderson y E. Cuhna. 2017. The effect of terrain on entheseal changes in the lower limbs. International Journal of Osteoarchaeology. DOI: 10.1002/oa.2597.

al-Oumaoui, I., S. Jiménez Brobeil y P. du Souich. 2004. Markers of activity patterns in some populations of the Iberian Peninsula. International Journal of Osteoarchaeology, 14: 343-359.

Alves Cardoso, F., C. Y. Henderson. 2013. The categorization of occupation in identified skeletal collections: a source of bias? International Journal of Osteoarchaeology, 23: 186-196.

Arrieta, M. A., O. J. Mendonça. 2011. Enfermedad degenerativa articular y uso del cuerpo en Rincón Chico 21 (Santa María, Catamarca). Revista Argentina de Antropología Biológica, 13 (1): 3-17.

Bonofiglio, M., N. de la Fuente y M. Herrera. 1982. Yacimiento arqueológico de Costasacate - Río Segundo - Córdoba. Actas y Memorias del VI Congreso Nacional de Arqueología Argentina. San Salvador de Jujuy.

Brooks, S. T., J. M. Suchey 1990. Skeletal age determination based on the os pubis: a comparison of the Acsádi-Nemeskéri and Suchey-Brooks methods. Human Evolution, 5: 227-238.

Buikstra, J., D. Ubelaker(eds.) 1994. Standards for Data Collection from Human Skeletal Remains. Field Museum of Natural History, Arkansas Archaeological Survey Research Series, N 44.

Fabra, M., C. González y S. Salega. 2012. Modos de vida e historia biológica de poblaciones de las Sierras y Llanuras de Córdoba (Argentina): aproximaciones desde el registro bioarqueológico. Revista Argentina de Antropología Biológica, 14: 87-104.

Fabra, M., S. Gordillo. 2014. Estimaciones acerca del uso de una almeja de agua dulce (Diplodonparallelopipedon) hallada en contexto arqueológico en el Mar de Ansenuza (Córdoba, Argentina). H. Hammond y $\mathrm{M}$. Zubimendi (eds), Arqueología y malacología: abordajes metodológicos y casos de estudio en el Cono Sur, 129144, VazquezMazzini Editores. Buenos Aires.

Hawkey, D., C. Merbs. 1995. Activity induced musculoskeletal stress markers (MSM) and subsistence strategy changes among ancient Hudson Bay Eskimos. International Journal of Osteoarchaeology, 5: 324-338. 
Henderson, C. Y. 2013. Subsistence strategy changes: the evidence of entheseal changes. Homo - Journal of Comparative Human Biology, 64: 491-508.

Henderson, C. Y., V. Mariotti, D. Pany-Kucera, S. Villotte y C. Wilczak. 2013. Recording Specific Entheseal Changes of Fibrocartilaginous Entheses: Initial Tests Using the Coimbra Method. International Journal of Osteoarchaeology, 23: 152-162.

Henderson, C. Y., V. Mariotti, D. Pany-Kucera, S. Villotte C. Wilczak. 2016. The new "Coimbra method": a biologically appropriate method for recording specific features of fibrocartilaginous entheseal changes. International Journal of Osteoarchaeology 26 (5): 925-932.

Jurmain, R. 2009. Understanding "musculoskeletal stress markers": their multifactorial etiology and constraints on simplistic interpretations. Workshop in Musculoskeletal Stress Markers (MSM): limitations and achievements in the reconstruction of past activity patterns. Coimbra, Portugal, 2-3 Julio 2009.

Jurmain, R., F. Alves Cardoso, C. Henderson y S. Villotte. 2012. Bioarchaeology's Holy Grail: the reconstruction of activity. A.L. Grauer (ed.) A Companion to Paleopathology, 12, 531-552, Wiley-Blackwell, Chichester.

Laguens, A. 1999. Arqueología del contacto hispanoindígena. Un estudio de cambios y continuidades en las Sierras Centrales de Argentina. BAR International Series 801. Oxford.

Laguens, A., M. Bonnin. 2009. Arqueología de Córdoba. Las sociedades indígenas de las Sierras Centrales de Argentina. Editorial UNC. Córdoba.

Larsen, C. S. 2002. Bioarchaeology. Interpreting behavior from the human skeleton. Cambridge University Press. Cambridge.

Lieverse, A., A. Weber, V. Bazaliiskiy, O. Goriunova y N. Savel'ev. 2007. Osteoarthritis in Siberia's Cis-Baikal: skeletal indicators of hunter-gatherer adaptation and cultural change. American Journal of Physical Anthropology, 132: 1-16.

Lieverse, A., V. Bazaliikii, O. Goriunova y A. Weber. 2013. Lower Limb Activity in the Cis-Baikal: Entheseal Changes Among Middle Holocene Siberian Foragers. American Journal of Physical Anthropology, 150 (3): 421-432.

Lovejoy, C. O., R. S. Meindl, T. Pryzbeck y R. P. Mensforth. 1985. Chronological metamorphosis of the auricular surface of the illium: a new method for the determination of age at death. American Journal of PhysicalAnthropology, 68: 15-28.
Medina, M., S. Pastor. 2012. Zooarqueología de sitios residenciales tardíos de las sierras de Córdoba (Argentina, ca.1100-300 AP): avances y perspectivas. Acosta, A., Loponte, D. y Mucciolo, L. (Eds) Temas de arqueología: estudios tafonómicos y zooarqueológicos II. INAPL. Buenos Aires.

Medina, M., S. Pastor y A. Recalde. 2016. The archaeological landscape of Late Prehispanic mixed foraging and cultivation economy (Sierras of Cordoba, Argentina). Journal of Anthropological Archaeology, 42: 88-104.

Milella, M., M. G. Belcastro, C. P. E. Zollikofer y V. Mariotti. 2012. The effects of age, sex, and physical activity on entheseal morphology in a contemporary Italian skeletal collection. American Journal of Physical Anthropology, 148: 379-388.

Michopoulou, E., E. Nikita y C. Y. Henderson. 2016. A test of the effectiveness of the Coimbra method in capturing activity-induced entheseal changes. International Journal of Osteoarchaeology DOI: 10.1002/oa.2564.

Pastor, S., E. Berberián. 2007. Arqueología del sector central de las Sierras de Córdoba (Argentina). Hacia una definición de los procesos sociales del período prehispánico tardío (900-1573 DC). Intersecciones en Antropología, 8: 31-47.

Pastor, S., L. López. 2010. Consideraciones sobre la agricultura prehispánica en el sector central de las Sierras de Córdoba. Korstanje, A. y Quesada, M. (Eds) Arqueología de la agricultura: casos de estudio en la región andina argentina. Editorial Magma. Tucumán.

Quevedo Kawasaki, S. 2000. Patrones de actividad a través de patologías en población arcaica de Punta Teatinos, Norte Semiárido chileno. Chungará, 32 (1): 7-9.

Salega, S. 2016. "Prácticas cotidianas, niveles de actividad y modos de vida en poblaciones prehispánicas del sector austral de las Sierras Pampeanas. Una aproximación bioarqueológica". Facultad de Filosofía y Humanidades, Universidad Nacional de Córdoba, Argentina, 276 pp. Tesis doctoral. Biblioteca de la Facultad de Filosofía y Humanidades.

Salega, S., M. Fabra. 2013. Niveles de actividad física en poblaciones de las Sierras y las Llanuras de la provincia de Córdoba (Argentina) durante el Holoceno tardío. Relaciones de la Sociedad Argentina de Antropología, 38 (2): 401-420.

Villotte, S. y C.J. Knüsel. 2013. Understanding Entheseal Changes: Definition and Life Course Changes. International Journal of Osteoarchaeology, 23: 135-146. 